\title{
A NEW METHOD FOR SCORING ACTIVE SWEAT GLANDS
}

David S. Gomez, Urio Mariani, Adolfo A. Leirner, Marcelo Mazzetto, João R. Sampel, and Marcus C. Ferreira

Two different and interrelated steps are involved in the assessment of active sweat glands: stimulation of sweating and its subsequent recording and quantification.

Sweat activation has been promoted by simple thermal stimulation, by intradermal injection of drugs such as methylcholine, ${ }^{1}$ and by dermal introduction of such drugs using iontophoresis. ${ }^{2,3}$ Gibson and Cooke's device for iontophoresis, one of the most employed devices, ${ }^{2}$ provides DC current and uses large lead electrodes lined with porous material. The positive pole is dampened with $2 \%$ pilocarpine hydrochloride, and the negative one with $0.9 \% \mathrm{NaCl}$ solution.

In order to visualize sweat glands, 2 methods have been used, ie, the starch-iodine test ${ }^{1,4}$ and a method that allows permanent recording of sweat drops on silicone. ${ }^{5}$ The latter method consists of stimulating sweat production, drying out the skin, and then applying a thin layer of liquid silicone to it. Prior to utilization, the silicone is mixed with a catalyzer that polymerizes and solidifies the material in about 90 seconds. The solid silicone is then manually removed, and sweat drops are counted by prints left on the material.

With respect to recording sweating activity, the methods reported in literature are limited to what was available in 1972 , as reviewed in a study by Harris et $\mathrm{al}^{5}$ and reiterated in the recent Rook's Textbook of Dermatology (2004), ${ }^{6}$ in which is stated "...silicone impression techniques are probably the most reliable...”.

Division of Burns and Plastic Surgery, Hospital das Clínicas, São Paulo University Medical School - São Paulo/SP, Brazil.

Heart Institute (InCor),Hospital das Clínicas, São Paulo University Medical School - São Paulo/SP, Brazil.

Plastic Surgery Division, Hospital das Clínicas, São Paulo University Medical School - São Paulo/SP, Brazil.

Email: davgomez@usp.br
We introduce a new dynamic operational method that includes 3 devices: 1) a small battery-powered iontophoretic apparatus to stimulate sweating, 2) a digital photo or video camera to record sequential digital photographs of sweat production, and 3) a computer loaded with digital image-processing software to quantitate the sweat drop production.

\section{TECHNIQUE}

A portable, small $(15 \times 15 \times 6 \mathrm{~cm})$ iontophoresis device (Figure 1) was designed and built by the Bioengineering Division of the Heart Institute InCor, São Paulo, Brazil. On its control panel, a knob sets the DC current applied to the skin, and an ammeter displays its value. Internal batteries power it. The electronic diagram of this device is shown in Figure 2. Stainless steel electrodes that are smaller than usual $\left(6-10 \mathrm{~cm}^{2}\right)$ were manufactured for our application. Porous tissue dampened with $2 \%$ pilocarpine hydrochloride and with saline are respectively

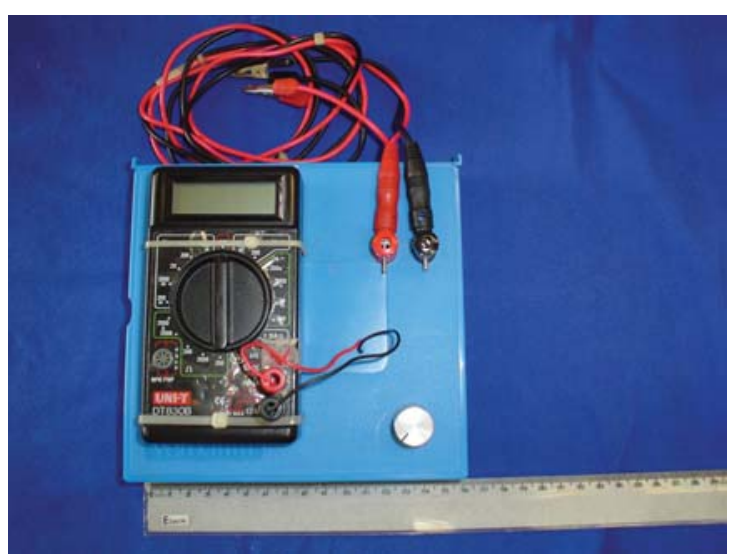

Figure 1 - Small iontophoresis device 


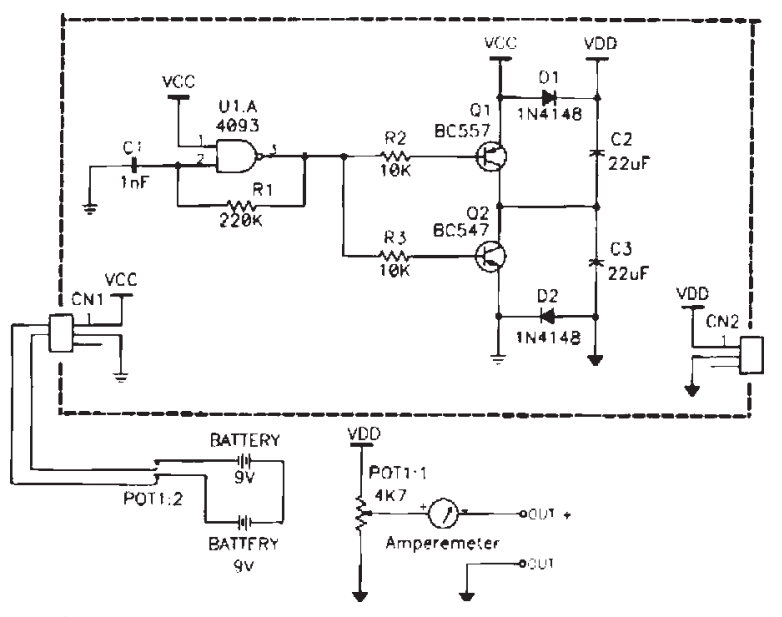

Figure 2 - Electronic diagram of the small iontophoresis device

placed between the positive and the negative electrodes and the skin. This device systematically induced sweating activity lasting for 30 minutes following the application of $2.5 \mathrm{~mA} \mathrm{DC}$ for 5 minutes. As soon sweating begins, the skin is dried, and recording is immediately started and carried out until drops flow together.

In order to record sweat drop images (Figure 3), a digital photo or video camera can be used. The digital videos or photographs (recorded for our study with a Nikon digital photo camera) are computer-processed using picture analysis software (ie, Image Tools) that counts the sweat drops in a given area over a given time interval.

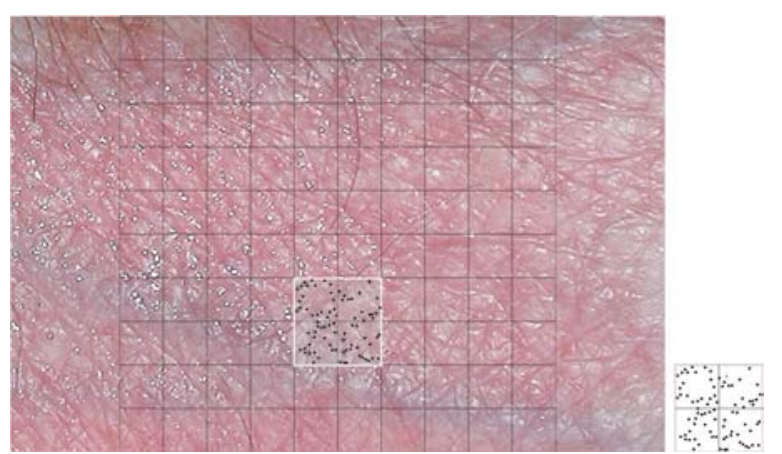

Figure 3 - Image of sweat drops emerging from skin taken by a Nikon digital photo camera and processed using the Image Tools software
Our tests have confirmed the efficacy of the device and safety of the method. The system for data processing regarding sweat gland activity allows good visualization, digital video and/or photographic recording, and quantification of both the emergence, growth and confluence of sweat drops, which appear as bright white points over the skin (Figure 3).

\section{DISCUSSION}

Our new technology for quantifying the activity of sweat glands improves on existing options in several ways. Compared to the Gibson and Cook device, our device is more compact so it is easier to find an appropriate place to store it and set it up for operation; it is battery powered so an electrical outlet is not necessary for its operation; additionally, a smaller sweat volume is needed, so the electrodes can be smaller. Compared to the recording and quantification of active sweat glands by drop printing on the silicone layer, our system avoids the problems involved achieving standardized mixtures of the silicone-catalyzer polymer that can result in different polymerization times, with consequent irregularities in sweat drop printing for each evaluated sample, resulting in a loss of accuracy. Additionally, only a static image of sweat production is provided with the silicone method-one obtained at the time of silicone polymerization, whereas the sweating process can be dynamically sampled using our method.

It is important to carry out a dynamic and continuous assessment of the development and growth of sweat drops until they flow together to make sure drops were produced by a single gland. Other methods aiming to score active sweat glands are unable to ensure that the drop comes from a single gland.

Our new system has additional advantages over other available technologies for quantifying the activity of sweat glands. The iontophoretic device activates sweat glands, and the accuracy of the dynamic record is higher.

The use of this new system for scoring the activity of sweat glands should increase the accuracy and precision of future studies that are concerned with sweating.

\section{REFERENCES}

1. Muller SA, Kierland RR. The use of a modified starch-iodine test for investigating local sweating responses to intradermal injection of methacholine. J Invest Dermatol. 1959;32:126.

2. Gibson LE, Cooke RE. A test for concentration of electrolytes in sweat in cystic fibrosis of the pancreas utilizing pilocarpine by iontophoresis. Pediatrics. 1959;7:545.

3. Kassan DG, Lynch AM, Stiller MJ. Physical enhancement of dermatologic drug delivery: Iontophoresis and phonophoresis. J Am Acad Dermatol. 1996;34:657.
4. Sato KT, Richardson A, Timm DE, Sato K. One-step iodine starch method for direct visualization of sweating. Am J Med Sci 1988;295:528.

5. Harris DR, Polk F, Willis I. Evaluating sweat gland activity with imprint techniques. J Invest Dermatol. 1972;58:78.

6. Coulson IH. Disorders of sweat glands. In: Burns T, Breathnach S, Cox N, Griffiths C, editors. Rook's Textbook of Dermatology. 7th ed. Massachusetts: Blackwell; 2004. p. 45-5. 